\title{
Electropherograms of reptile species commonly kept as pets
}

\author{
Christoph Leineweber, Rachel E. Marschang \\ LABOKLIN GmbH \& Co. KG, Bad Kissingen, Germany \\ Contact: c.leineweber@laboklin.com
}

\section{Introduction}

Electrophoresis is often used for the detection of changes in the blood protein composition caused by different diseases, especially in mammals and birds. This technique is also being increasingly used in reptile medicine. The goal of this presentation is to demonstrate typical electropherograms of capillary zone electrophoresis (CZE) of 8 reptile species commonly kept as pets in Europe.

Fig. 1 Hermann's tortoise

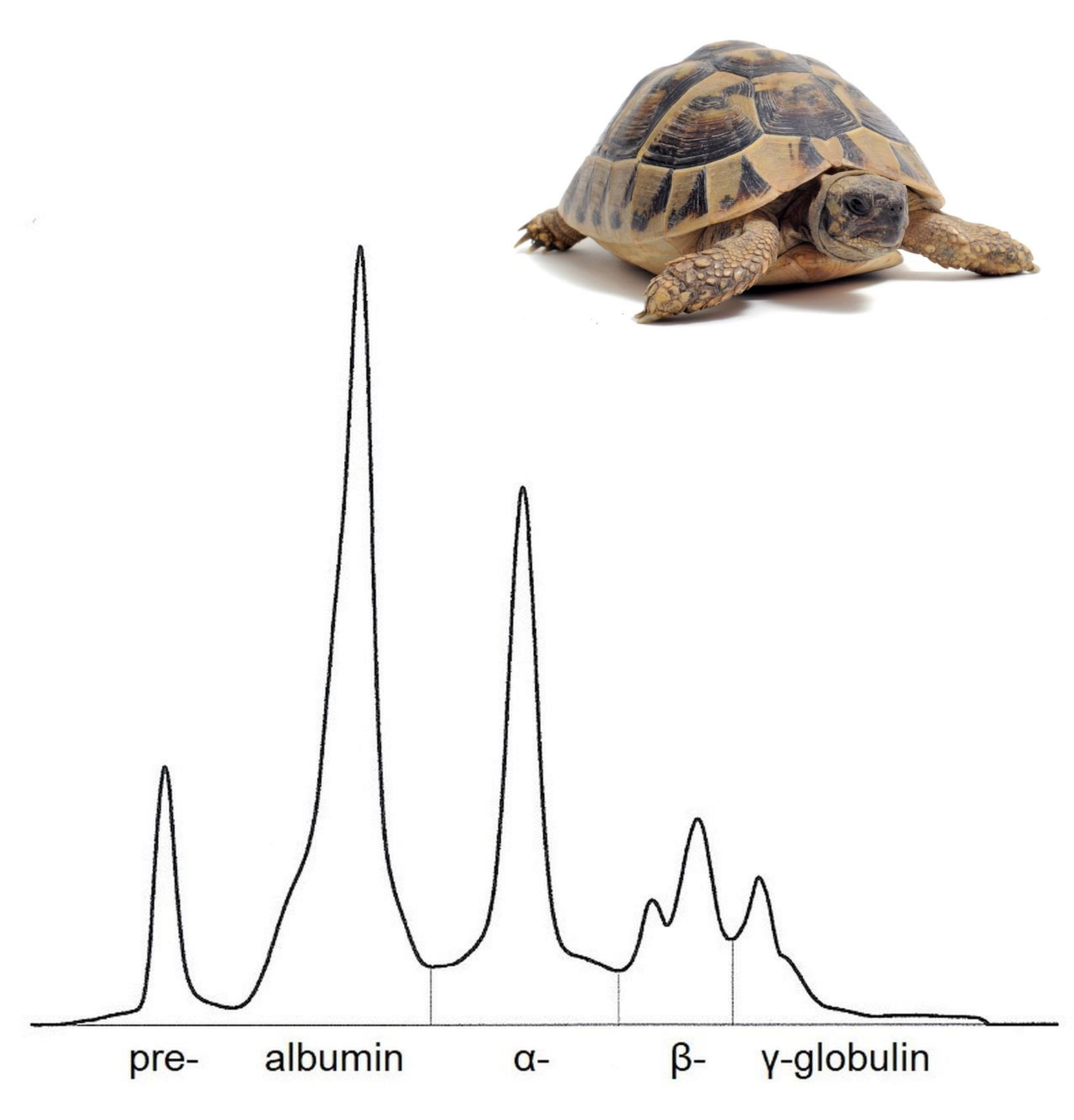

Fig. 2 Spur-thighed tortoise

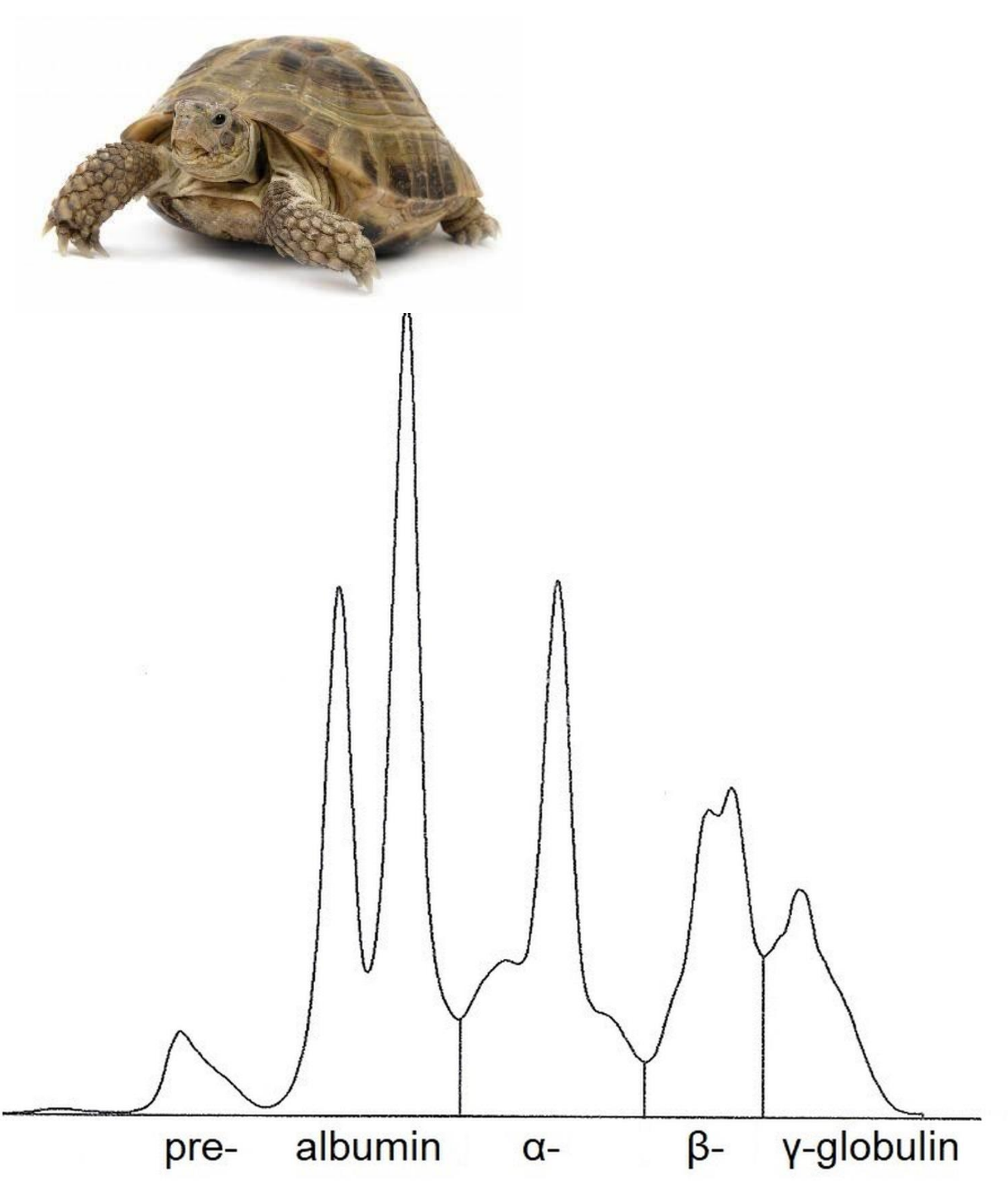

Fig. 3 Red-eared slider
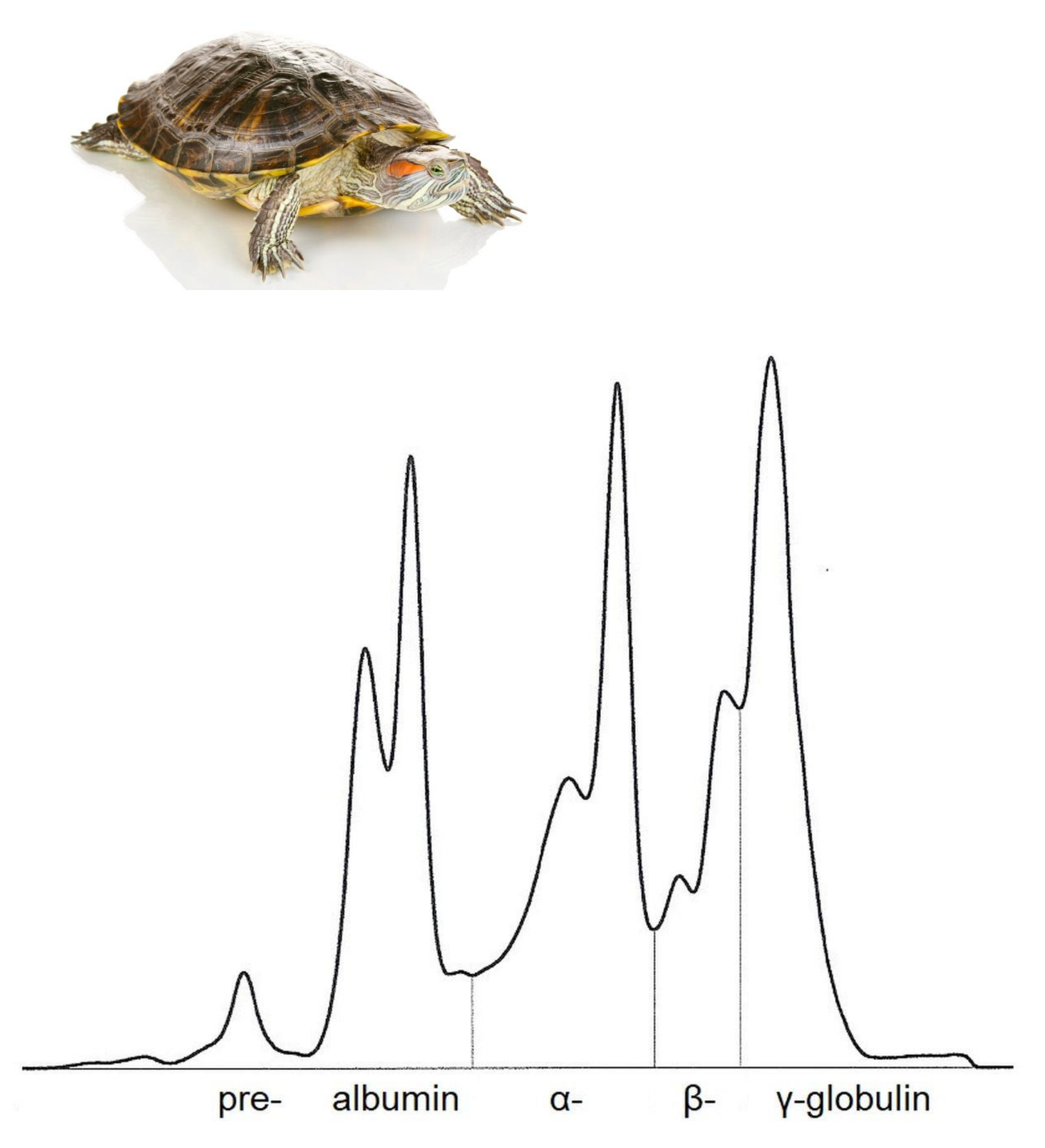

Fig. 4 Bearded dragon

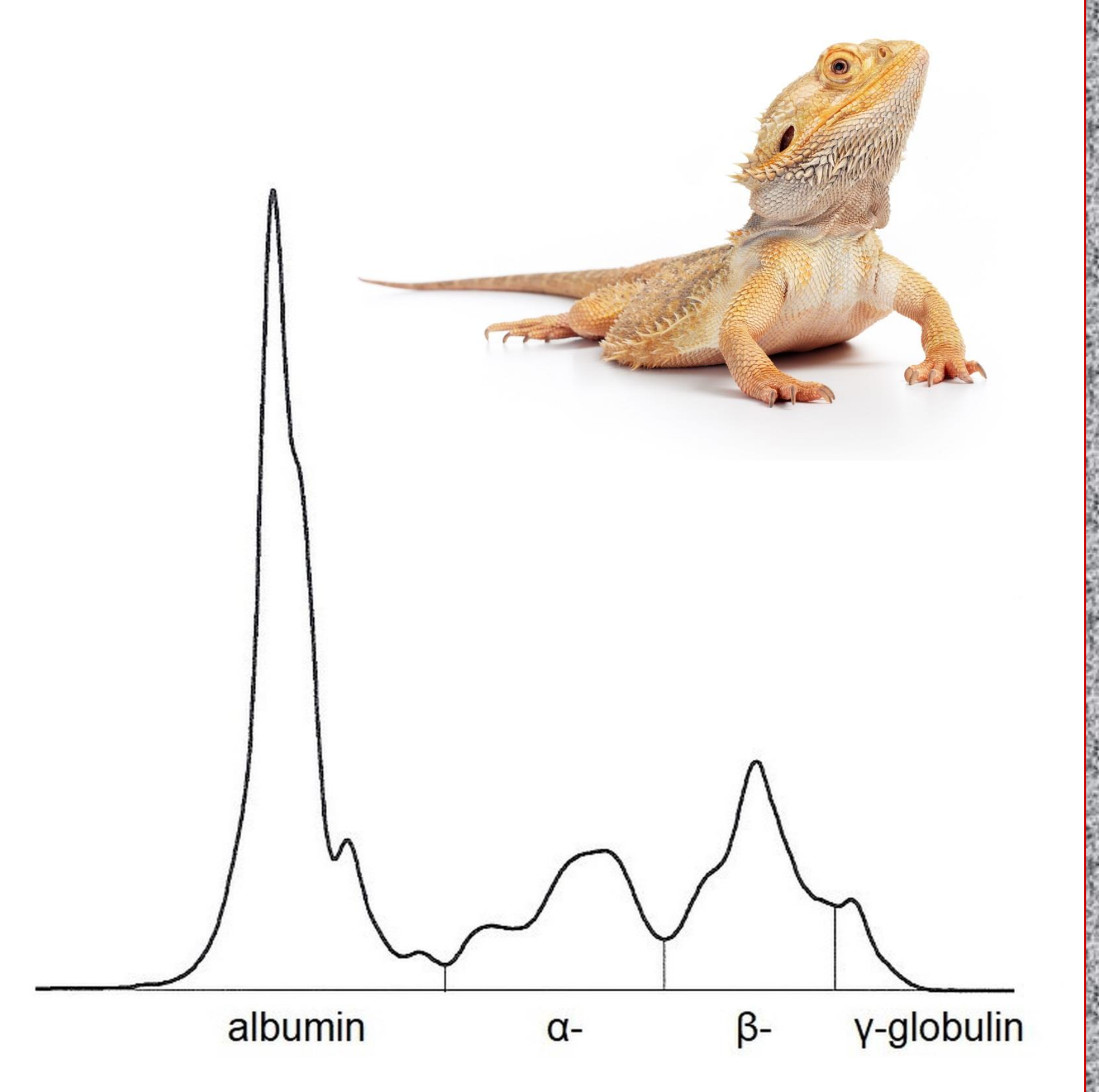

Materials and Methods

- Lithium heparinized plasma samples were analysed from:

- Hermann's tortoise (Testudo hermanni)

- Spur-thighed tortoise (Testudo graeca)

- Red-eared slider (Trachemys scripta elegans)

- Bearded dragon (Pogona vitticeps)

- Panther chameleon (Furcifer pardalis)

- Boa constrictor (Boa constrictor)

- Ball python (Python regius)

- Corn snake (Pantherophis guttatus)

- The samples were collected for routine health screening and a clinical chemistry profile was obtained from all samples

- All animals were clinically healthy

- Total protein was measured by the biuret method using the cobas@ 8000 analyzer series (Roche Diagnostics, Germany)

- Plasma electrophoresis was performed by CZE using the Sebia MINICAP (Sebia, Lissex, Evry Cedex, France)

\section{$\underline{\text { Results }}$}

- Representative electropherograms are shown in figures 1-8

- Variations between the different reptile species are visible in different numbers und distances between the protein peaks

- Some have a prealbumin fraction

- In comparison to the other reptiles tested, chelonians had larger a-globulin fractions

- Squamates had smaller globulin peaks in comparison to the turtles

- Snakes had larger $\beta$-globulin fractions
Fig. 6 Boa constrictor

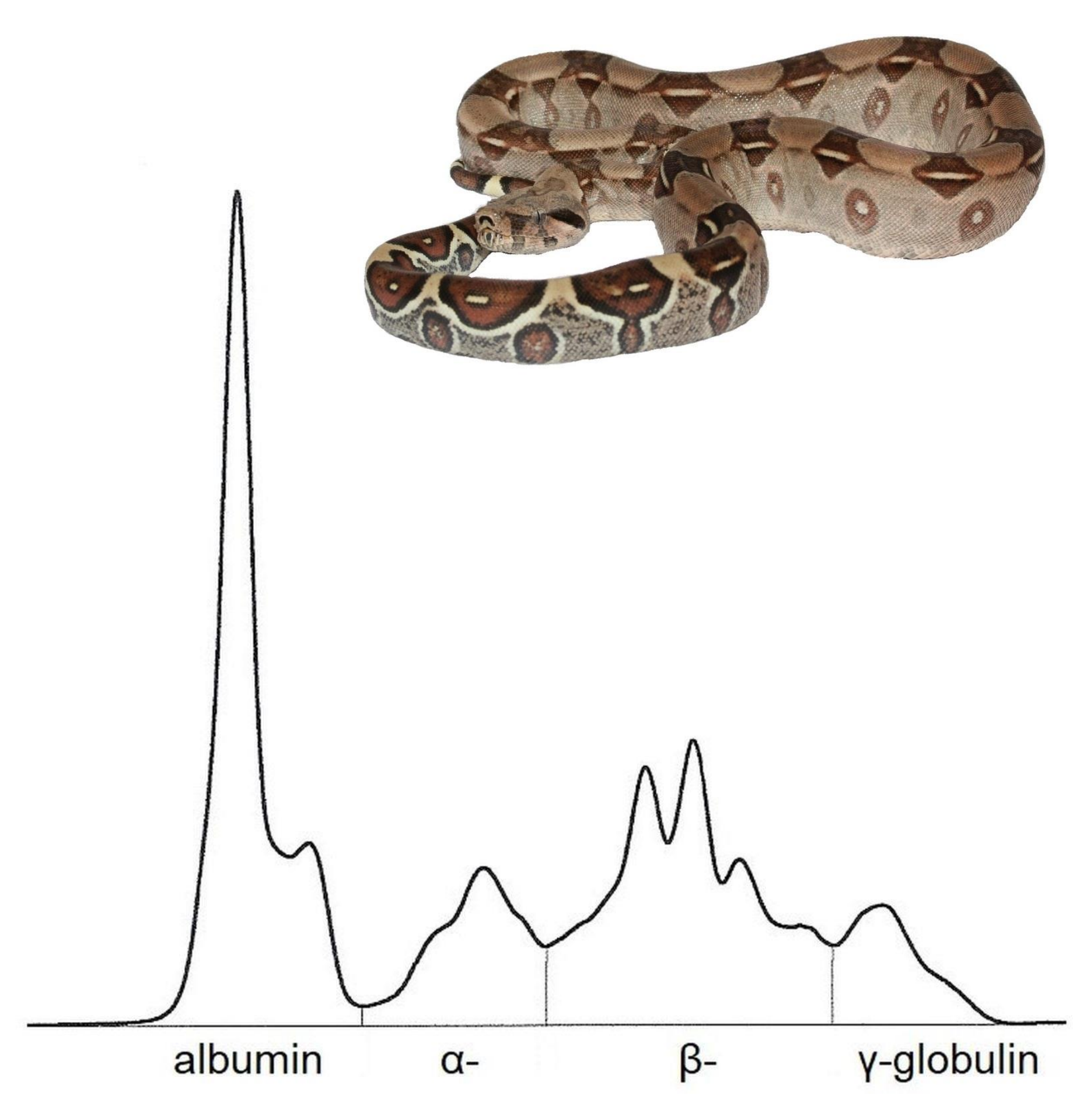

Fig. 7 Ball python

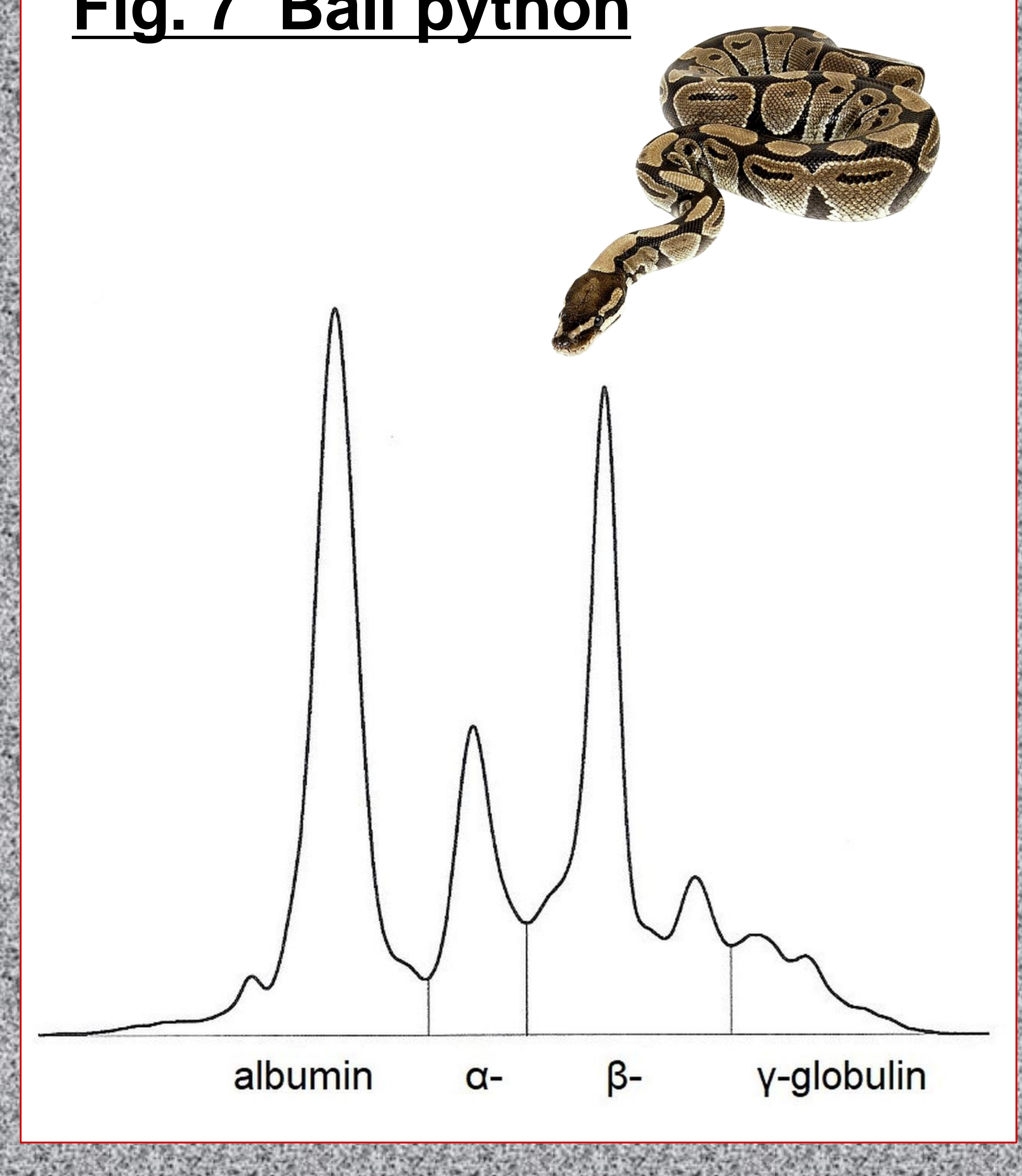

Fig. 8 Corn snake

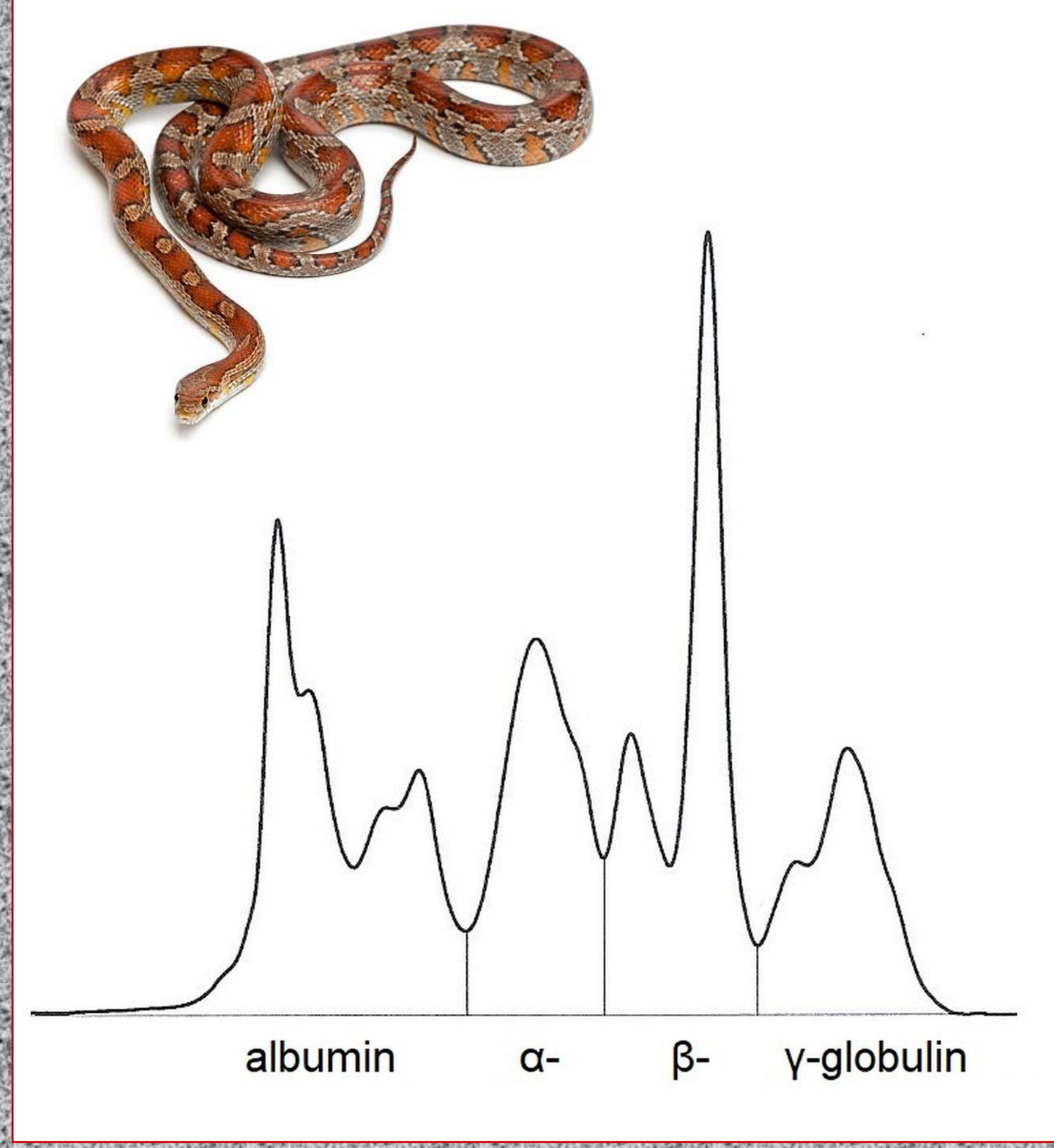

\section{Impact and Clinical Significance}

Electropherograms can be used as a tool for health assessment in reptiles, but evaluation must be based on species specific parameters. Further studies are necessary for the establishment of reference intervals in healthy individuals and for the diagnosis of specific protein changes due to frequently presented infectious diseases like reptarenavirus infections in snakes or herpesvirus infections in chelonians. 